\title{
El papel de la genética en la aparición y desarrollo de la periodontitis. II: Polimorfismos asociados a la enfermedad periodontal
}

\author{
RODRIGO-GÓMEZ D* \\ OTEO-CALATAYUD A** \\ ALONSO-ROSADO A $* * *$ \\ BASCONES-MARTÍNEZ $\mathrm{A} * * * *$
}

\begin{abstract}
Rodrigo-Gómez D, Oteo-Calatayud A, Alonso-Rosado A, BasconesMartínez A. El papel de la genética en la aparición y desarrollo de la periodontitis. II: Polimorfismos asociados a la enfermedad periodontal. Av Periodon Implantol. 2008; 20, 2: 121-130.
\end{abstract}

\section{RESUMEN}

La periodontitis es una enfermedad multifactorial que resulta de la interacción de bacterias periodontopatógenas con los mecanismos de respuesta inmune del huésped y que se caracteriza por una reacción inflamatoria que afecta al aparato de inserción del diente. Las enfermedades multifactoriales habitualmente envuelven complejas interacciones de muchos genes y factores ambientales. El tipo de variaciones genéticas involucradas en este tipo de enfermedades se denominan polimorfismos genéticos, son varios y normalmente sus efectos fenotípicos son limitados.

En la primera parte de esta revisión analizamos la evidencia científica de la asociación entre periodontitis y genética. En esta segunda parte revisaremos esta asociación fundamentalmente desde las investigaciones sobre los polimorfismos con una proyección más relevante en su relación con la periodontitis.

\section{PALABRAS CLAVE}

Periodontitis, susceptibilidad, genética, polimorfismos.

Fecha de recepción: Noviembre 2007

Aceptado para publicación: Diciembre 2007

\section{INTRODUCCIÓN}

En humanos, los estudios sobre las variaciones hereditarias en el sistema inmune y la periodontitis son, como ya comentamos en la primera parte de esta revi- sión, muy complejos. De hecho, frecuentemente, el fenotipo observado es el resultado de una gran cantidad de influencias genéticas y ambientales. Esta circunstancia cobra especial relevancia con las defensas del huésped, las bacterias gram-negativas y lipopoli-

* $\quad$ Odontólogo por la U.E.M. Máster en periodoncia e implantes por la U.C.M.

** Odontólogo por la U.E.M. Máster en periodoncia e implantes por la U.C.M.

*** Doctor Médico-Estomatólogo. Universidad de Salamanca. Máster en Periodoncia UCM. Profesor Asociado. Facultad de Odontología de Salamanca. USAL.

**** Catedrático de Medicina Bucal y Periodoncia. F. de Odontología U.C.M. 
sacáridos en cuya interacción se ven envueltos gran cantidad de factores moleculares y celulares. La búsqueda de marcadores genéticos asociados con la severidad, y susceptibilidad a la periodontitis está recibiendo actualmente una gran atención. En particular, los polimorfismos de genes que codifican moléculas relacionadas con el sistema inmune del huésped.

Algunos factores de la respuesta inmune se han asociado con diferentes formas clínicas de periodontitis; para algunos de estos factores los determinantes genéticos subyacentes son conocidos. Es probable que los polimorfismos de estos determinantes tengan un papel importante en las distintas susceptibilidades a la enfermedad periodontal y su comprensión pueda ayudarnos a entender los diferentes patrones de susceptibilidad. La clave será identificar factores genéticos suficientemente importantes para conferir un riesgo clínico significativo. En general un gen puede ser considerado como un candidato para un papel causal o modificador de la periodontitis si el proceso fisiológico determinado por dicho gen se ha asociado con el inicio, progresión o severidad de la enfermedad periodontal (1).

A continuación se detallan los polimorfismos más estudiados y con una proyección más relevante en su relación con la periodontitis

\section{GENES Y POLIMORFISMOS GENÉTICOS IMPLICADOS EN LA PERIODONTITIS}

\section{HORIMONAS}

VITAMINA D: Los polimorfismos genéticos en el receptor de la vitamina D están asociados con la homeostasis del hueso y las enfermedades relacionadas con la pérdida de este tejido (1). Hay estudios que sugieren que la osteopenia podría ser un factor predisponente en la periodontitis al incrementar la susceptibilidad a los efectos de determinados mediadores de pérdida ósea (3). La etiología de la osteopenia generalizada es multifactorial y comparte diversos factores de riesgo con la periodontitis como el tabaco (3). Existen estudios que indican que la osteopenia y la pérdida de hueso sistémica se relacionan con la pérdida de hueso alveolar, inserción clínica y dientes (4 y 5 ).

La vitamina $\mathrm{D}$ expresa su acción genómica por vía de su receptor (VDR), el cual muestra numerosos polimorfismos. Este receptor se expresa en varios tipos celulares como los linfocitos y macrófagos habiéndose observado que el alelo "b" del gen del receptor VDR esta asociado a una mayor densidad ósea (6). Algunos estudios han relacionado determinados polimorfismos del receptor de la vitamina $\mathrm{D}$ con periodontitis agresiva (2), especialmente en conjunción con otros polimorfismos (7). Otros trabajos han encontrado una relación similar con la periodontitis crónica. Se ha observado que la expresión de determinados alelos del VDR pueden hacer a los pacientes 2.4 veces más susceptibles a la enfermedad que pacientes con ausencia de ese alelo. Estos hallazgos indican que el genotipo del receptor VDR puede ser un indicador de riesgo para la susceptibilidad a la periodontitis.

\section{DISFUNCIÓN DE LOS NEUTRÓFILOS}

Los neutrófilos polimofonucleares (PMN) constituyen la mayor parte del componente celular de la respuesta inmune celular innata, especialmente contra infecciones bacterianas. Algunos defectos genéticos que afectan a la función de los neutrófilos se sabe que predisponen a infecciones microbianas como la periodontitis (8).

En el surco gingival los PMNs son el pilar en la defensa de bacterias patógenas. Los PMNs emigran de la sangre periférica a los lugares de extravasación. Determinadas sustancias bacterianas actúan como quimioatrayentes hacia estas localizaciones.Se han descrito defectos en la quimiotaxis en algunas enfermedades o síndromes como el Síndrome de ChédiacHigashi, diabetes mellitus, síndrome de Papillon-Lefevre o en algunas formas de periodontitis de comienzo temprano. Una proporción substancial de pacientes con periodontitis juvenil localizada (alrededor del 70\%) tienen algún tipo de defecto en la habilidad de los neutrófilos para responder ante agentes quimiotácticos.

Una proporción significativa de la variación interindividual de la respuesta inmune a agresiones bacterianas parece estar bajo control genético. Variaciones genéticas que produzcan alteraciones en la función de los neutrófilos, principalmente en la expresión de sus receptores de superficie, son candidatas razonables como determinantes genéticos para un incremento de la susceptibilidad a la periodontitis (9)

\section{INIMUNOGLOBULINAS}

La respuesta sérica de las inmunoglobulinas frente a algunos microorganismos es un determinante clave en 
el control y limitación de infecciones bacterianas. Sabemos que diferentes subclases de inmunoglobulinas tienen diferentes funciones en la defensa del huésped. El déficit de la inmunoglobulina (IgG), por ejemplo, está asociada a infecciones recurrentes durante la infancia (10). Éste tipo de inmunoglobulina esta fuertemente influenciada por determinadas citoquinas y por tanto, puede afectarse por variaciones genéticas de las mismas. (11). Por otro lado sabemos que algunas sustancias como el tabaco son capaces de reducir los niveles séricos de IgG (12) Por tanto junto a los factores inherentes al huésped, existen factores ambientales capaces de modular la respuesta del tipo IgG (12).

La inmunoglobulina IgG2 es la primera que reacciona con los carbohidratos y polisacáridos bacterianos y es la dominante tanto en la periodontitis de comienzo temprano como en la del adulto. Los datos provenientes de algunas investigaciones (13) apoyan la hipótesis de que un incremento sérico de IgG2 podría proporcionar suficiente protección contra la progresión de bacterias periodontopatógenas como el Aa. Esta circunstancia daría lugar a una forma localizada, en vez de generalizada de la periodontitis de comienzo temprano.

Las moléculas IgG contienen variaciones genéticas en sus dos cadenas; la cadena pesada gamma, denominados alotipos $\mathrm{Gm}$, y cadena ligera kappa, denominada alotipo $\mathrm{Km}$. Aunque para otras IgG se conocen varios alotipos, el único identificado para la IgG2 es el G2m (n) (14). Los genes responsables de estos alotipos parecen influenciar la expresión y por tanto la respuesta de la IgG2 (13). Esta respuesta es específica para cada raza (15). Aproximadamente dos de cada tres caucásicos son positivos para el alotipo G2m (14) y sabemos por ejemplo que los jóvenes de raza caucásica con una baja expresión fenotípica de la inmunoglobulina G2m (23) tienen una mayor predisposición a infecciones por bacterias específicas.

La eficacia fagocítica óptima de los anticuerpos del tipo IgG2 requiere de la presencia de neutrófilos que expresen receptores de superficie del tipo Fc capaces de reconocer este anticuerpo. Por tanto, la efectividad para proteger contra determinadas bacterias periodontopatógenas como el Aa o Pg está influenciada tanto por los niveles séricos y afinidad de la IgG2 como la expresión de receptores del tipo Fc en los neutrófilos, circunstancias estas determinadas genéticamente.

\section{RECEPTORES DE SUPERFICIE}

\section{a) RECEPTOR FC-GAMMA}

El funcionamiento normal de las células fagocíticas es esencial para la defensa del organismo frente a agentes microbianos. Las células fagocíticas en los seres humanos se comunican con el exterior a través de receptores de superficie. Los neutrófilos utilizan los receptores del tipo Fc para unirse a las inmunoglobulinas dando un paso crucial en la opsonización y fagocitosis de las bacterias. Cualquier cambio estructural debido a una variación genética en los receptores Fc puede alterar la habilidad de los neutrófilos para responder a complejos inmunes y eliminar la infección. Determinados polimorfismos en los receptores Fc expresados en la superficie de las células fagocíticas muestran un papel relevante en la susceptibilidad a infecciones. Los genes del receptor de superficie Fcgamma se alojan en el cromosoma 1 y codifican tres clases de receptor: El Fc-gammaRI (CD64), FcgammaRII (CD32) y Fc-gammaRIII (CD16). Estas clases se subdividen a su vez en subclases. Fc-gammaRIa y b, Fc-gammaRIIa, b y c y Fc-gammaRIII a y b. El receptor Fc-gamma RIIa (CD32) reconoce la fracción Fc de la IgG2 de modo que los neutrófilos que expresan dicho receptor son capaces de reconocer las bacterias que han sido opsonizadas por esta inmunoglobulina.

El gen del receptor FC-gamma RIIa o CD32 para inmunoglobulinas puede expresar 2 alelos, que difieren significativamente en su capacidad para unirse a la IgG2. Los dos alelos difieren por el aminoácido, arginina (R13l) o histidina (H13l). Aunque existen varios receptores del tipo Fc-gamma, el H13l es el único que reconoce de manera eficaz la IgG2, y la unión máxima a esta inmunoglobulina se presenta en los homocigotos para este receptor (H131). Individuos con una pobre afinidad del receptor CD32 pueden manifestar un aumento de susceptibilidad a infecciones.

Como vimos anteriormente, la respuesta de la IgG2 tiene un papel importante en la periodontitis. Aunque este tipo de IgG se ha visto tradicionalmente como una inmunoglobulina con una pobre capacidad de opsonización, trabajos reciente (16 y 17) muestran que la IgG2 si tiene una capacidad significativa de opsonización con fagocitos que expresan el receptor $\mathrm{H} 13 \mathrm{l}$. Wilson y col. 1997 (17) observaron que la IgG2 era significativamente más efectiva en la mediación de la fagocitosis del Aa, en conjunción con neutrófilos huma- 
nos homocigotos para el receptor $\mathrm{H} 13 \mathrm{l}$ cuando se comparaba con individuos homocigotos para el receptor R131.El polimorfismo genético que define el receptor Fc-gamma RIIa, por tanto, parece ser un marcador prometedor en susceptibilidad a determinadas formas de periodontitis. De hecho, algunos trabajos han constatado que el genotipo Fc-gamma RII H/H13l se hace especialmente influyente en la susceptibilidad y severidad a la periodontitis ante la presencia de otros factores de riesgo como el tabaco (7).

\section{b) ANTÍGENOS DEL SISTEMA MAYOR DE HISTOCOIMPATIBILIDAD (HLA)}

El sistema mayor de histocompatibilidad está constituido por un grupo de genes localizados en el cromosoma 6. La región HLA esta dividida en 3 regiones: La tipo I $(A, B, C)$, la tipo II(DR,DQ,DP) y la tipo III (Factores del complemento). Aunque la función clásica de los HLA clase I y II es el reconocimiento celular, se ha observado que pueden servir como marcadores de algunas enfermedades con base genética. Algunas de estas patologías que han mostrado una fuerte asociación positiva con fenotipos específicos del HLA son la espondilitis anquilosante, diabetes mellitus insulinodependiente $o$ la artritis reumatoide (18).

EL HLA DR esta involucrado en la presentación de antígenos y se cree que interviene en el mantenimiento de la actividad proliferativa de los linfocitos (19). Visón-Boutilliez y col. mostraron en un estudio un incremento en la expresión de HLA DR por células epiteliales en pacientes periodontales cuando se comparaba con sanos (11.2).

La mayor parte de los estudios se han centrado en la periodontitis de comienzo temprano y su asociación entre diferentes fenotipos HLA. Estas investigaciones han obtenido asociaciones positivas entre los antígenos A9 (20), B15 (21), A28 (21 y 22) y DR4 (23 y 24) con diferentes formas de PCT. Otros trabajos han mostrado asociaciones negativas con los antígenos A2 (25) y Al0 (20), mientras que otras investigaciones no han sido capaces de encontrar ningún tipo de asociación (25 y 26 ).

Es importante señalar a la hora de interpretar los resultados obtenidos por estos estudios las dificultades que surgen debido fundamentalmente a los diferentes criterios para definir la periodontitis de comienzo temprano y por las conocidas diferencias de los antígenos HLA entre diversos grupos étnicos.

\section{ENZIMMAS}

La actividad metabólica para sustancias foráneas por determinadas enzimas como la $\mathrm{N}$-acetiltransferasa determina la susceptibilidad individual para la toxicidad de ciertos fármacos, drogas o agentes químicos (62). Debido a un polimorfismo genético, los sujetos pueden clasificarse como acetiladores rápidos o lentos de acuerdo a como su organismo es capaz de metabolizar ciertas sustancias nocivas. En general los acetiladores lentos son más susceptibles a los efectos de ciertas drogas o sustancias como las presentes en el tabaco debido a su eliminación retardada y el consiguiente aumento de su concentración. En caucasianos la frecuencia de acetiladores lentos es del 55-60\% (27) $y$, por tanto, el impacto de este polimorfismo en determinadas enfermedades multifactoriales debe ser tenida muy en cuenta. Los trabajos que han investigado la relación de los diferentes polimorfismos de esta enzima y la periodontitis se han basado en la hipótesis de que pacientes con periodontitis y el polimorfismo que determina una acetilación más lenta son más susceptibles a los efectos nocivos del tabaco en el periodonto. Kocher y col evaluaron esta cuestión en 155 pacientes periodontales caucasianos divididos en tres grupos, uno con periodontitis moderada, otro severa y el tercero sin periodontitis, Los datos obtenidos en esta investigación y otra realizada por el mismo grupo (28) concluyeron que el fenotipo acetilador lento esta asociado con formas más severas de periodontitis. Los resultados de estos trabajos sugieren por tanto que los pacientes con un fenotipo que determina una acetilación más lenta en fumadores tienen mayor riesgo de padecer periodontitis.

\section{MEDIADORES DE LA INFLAMACIÓN}

\section{a) Prostaglandina E2}

Las prostaglandinas (PGD) son enzimas que actúan como potentes mediadores biológicos. Tienen efectos fisiológicos que participan en una gran variedad de procesos patológicos como la periodontitis. La enzima prostaglandina endoperoxidasa convierte el ácido araquidónico en prostaglandina $\mathrm{H} 2$ precursor de las prostaglandinas, protaciclinas y tromboxanos. Existen dos isoformas para la enzima, las prostaglandinas peroxidasa 1 y 2 , con similar actividad catalítica. La 1 es expresada en numerosos tejidos y parece que genera PGD involucradas en el mantenimiento de funciones fisiológicas esenciales. En contraste la 2 no aparece de forma constante, pero su expresión es inducida 
rápidamente ante estímulos proinflamatorios como citoquinas, factores de crecimiento o lipopolisacáridos bacterianos.

La PGD E2 se ha implicado en la patogénesis de la periodontitis del adulto y de comienzo temprano considerándose un potente mediador de gran parte de los procesos de destrucción tisular ocurridos durante la enfermedad periodontal (29 y 30 ). Se ha identificado la conexión de una región cromosómica (9q32-33) con la periodontitis de comienzo temprano (1). Esta región física incluye genes para la enzima prostaglandina endoperoxidasa 1. Estos hallazgos animan a realizar estudios que intenten asociar marcadores genéticos de la prostaglandina E2 con la clínica de la periodontitis.

\section{b) Citouinas}

\section{Interleuquinas}

\section{II-1}

El sistema inmune produce citoquinas y factores humorales para proteger los tejidos del huésped de los agentes inflamatorios, invasiones bacterianas y traumatismos. En la mayoría de ocasiones este sistema es capaz de devolver una homeostasis normal. Sin embargo en algunas situaciones, la sobreproducción de mediadores inflamatorios pueden causar daño al huésped.

El papel de las citoquinas en la patogénesis de la enfermedad periodontal ha sido objeto de estudio de numerosas investigaciones, particularmente la interleuquina 1. Las fases activas de la periodontitis producen un aumento de los niveles de las interleuquinas $l \alpha$ y $\beta$. La Il-1 predominante en los tejidos periodontales es la IL-1 1 y, debido a sus múltiples propiedades proinflamatorias, tiene un papel especialmente relevante en la patogénesis de esta infección. Algunos trabajos indican que los polimorfismos en el gen de la IL-1 influencian las variaciones en la síntesis de citoquinas, y esto tiene como consecuencia una modificación de la respuesta individual frente a estímulos bacterianos (31 y 32$)$.

El test genético de susceptibilidad periodontal (PST) (33) evalúa la ocurrencia simultánea del alelo 2 en los loci IL-1 $\alpha+4.845$ y IL-1 $\beta+3.954$. Se considera un paciente como IL-1 positivo cuando se encuentran presentes el alelo 2 de en la IL- $1 \alpha+4.845$ junto con el alelo 2 del polimorfismo +3.954 para el gen de la IL-1ß y se asocia con producciones elevadas para esta cito- quina. Investigaciones como la del grupo de Kornman y cols. (33) observaron un incremento del riesgo a padecer periodontitis severa 20 veces superior en pacientes de más de 40 años con un genotipo positivo.

Nieri y col. 2002 (34) encontraron una asociación entre la pérdida de hueso inicial y el polimorfismo de la IL-1 como indicador pronóstico para futura pérdida de hueso. Algunos estudios transversales muestran que los pacientes IL-1 positivos que no fuman tienen más riesgo de periodontitis avanzada en edades más tempranas que los pacientes IL- 1 negativos (35 y 36). Por su parte Meisel y col $(2002,2004)$ (37 y 38) observaron un riesgo 4 veces superior de pérdida de inserción para pacientes IL-1 positivos y fumadores en comparación con no fumadores. Estos trabajos demuestran una interacción evidente entre ambiente y genes y coinciden con los obtenidos para pacientes en mantenimiento por McGuire y Nunn (1999) (39). En este estudio, tras un seguimiento de 14 años observaron un mayor riesgo de pérdida de dientes en pacientes fumadores y positivos para la IL-l. Los pacientes con un genotipo positivo para la IL-1 incrementaban el riesgo de pérdida de dientes 2,7 veces y los grandes fumadores 2,9 veces. La combinación de ambas circunstancias incrementaba el riesgo de pérdida de dientes hasta 7,7 veces. A pesar de estas y otras investigaciones ( 40 y 41 ) el papel del genotipo de la IL- 1 es controvertido y a veces inconsistente (Taylor 2001). En un estudio prospectivo a 5 años (42) con 295 pacientes, los autores concluyeron que el genotipo de la IL-1 era un factor de riesgo que contribuía pero no de forma determinante en la progresión de la periodontitis. Otros estudios no han encontrado ningún tipo de asociación para este factor ni a corto (43) ni a largo plazo (63). En relación a estos resultados dispares es importante entender la variabilidad étnica en la prevalencia de este genotipo. (44) Por ejemplo en China se ha encontrado una prevalencia positiva para el genotipo de la IL-1 en el $2,3 \%$ de los sujetos (45), en Europeos un rango de entre el 29 y el $46 \%$ y en Americanos caucasianos de entre el 29 y el 38\% (41). Los resultados contradictorios pueden explicarse por otros factores como las diferencias en los criterios diagnósticos, el número de sujetos estudiados o las características de los grupos control (41).

\section{Il-4}

Esta interleuquina con propiedades antiinflamatorias es un importante regulador de la función de los macrófagos y es capaz de inhibir la secreción de PGE2 y otras citoquinas producidas por estas células. Ésta in- 
terleuquina puede inhibir el receptor CDl4 (46) y se ha demostrado que induce la apoptosis en monocitos. También se ha demostrado que inhibe la producción de algunas metaloproteinasas mediadas por la IL-1 (47) Algunas de las últimas investigaciones muestran que el $27.8 \%$ de los paciente con periodontitis de comienzo temprano son positivos para determinados polimorfismos de la IL-4 (48) otras investigaciones en periodontitis agresiva no han encontrado asociación (49).

\section{II-10}

Es una citoquina con capacidad de inhibir la síntesis de citoquinas proinflamatorias. Algunos estudios en animales han observado que su disminución agrava la reabsorción ósea (50) El gen responsable de esta interleuquina se encuentra ubicado en el cromosoma l y se han descrito 3 polimorfismos bialélicos diferentes dentro de la región promotora: GCC, ACC y ATA. El GCC se ha asociado con una producción mayor de IL-10 (51) sugiriendo que las otras dos variaciones genéticas deben exhibir una respuesta inflamatoria más acusada que la variante GCC. Aunque la presencia de una asociación entre polimorfismos del gen de la IL-10 y manifestaciones clínicas de enfermedades inflamatorias crónicas como la periodontitis es controvertido, algunos trabajos como el de Scarel-caminaga y col (2004) (52), en población brasileña o el de Berglundh y col. (2003) (53) en suiza, han encontrado asociaciones positivas para determinados polimorfismos de la IL-10 y un incremento de la susceptibilidad a la periodontitis.

\section{Factor de crecimiento transformador TGF-ß1}

Este factor de crecimiento representa una familia de polipéptidos involucrados en la inflamación y regulación de la respuesta inmune. Pocos estudios han investigado la relación entre la periodontitis y el polimorfismo de este factor de crecimiento. Los trabajos existentes no han podido confirmar que el polimorfismo del gen del TGF- $ß 1$ influencie la severidad o susceptibilidad a la periodontitis del adulto (54) o han demostrado un efecto muy limitado.

\section{Factor de necrosis tumoral alfa TNF- $\alpha$}

Existen argumentos para pensar que el gen del TNF- $\alpha$ es un buen candidato para estudios genéticos en relación con la periodontitis; esta citoquina es un potente mediador inmunológico con propiedades proinflamatorias y posee capacidad para incrementar la reabsorción ósea (55).

Se han observado diferencias interindividuales en la producción de este factor por parte de células sanguíneas mononucleares y leucocitos orales. Es comprensible plantear la hipótesis de que algunas de las diferencias individuales en la susceptibilidad o severidad de la periodontitis sean debidas a diferencias determinadas genéticamente en la producción de este factor. Los alelos del TNF- $\alpha$ se han propuesto como marcadores genéticos para la enfermedad periodontal, especialmente la del adulto. Los niveles de este factor están aumentados en el fluido crevicular de tejidos periodontales inflamados con respecto a pacientes sanos (56). Sin embargo, hasta la fecha tenemos datos confusos al respecto, existen investigaciones con resultados a favor de la relación entre polimorfismos del TNF- $\alpha$ y la periodontitis (57 y 58) así como en contra de dicha relación (59 y 60).

\section{Factor de necrosis tumoral beta (TNF-ß)}

El gen del TNF- $ß$ está relacionado íntimamente con el gen del TNF- $\alpha$ en la misma región cromosómica. El TNFB está expresado por linfocitos CD-4, células natural killer y células B activadas (61). Algunas investigaciones han relacionado los polimorfismos de esta citoquina con la susceptibilidad a la periodontitis del adulto, especialmente cuando interacciona con los polimorfismos de otros mediadores como el de la enzima convertidora de la angiotensina (ACE) o la endotelina-1 (ET-1) (54).

\section{IMPLICACIONES CLÍNICAS Y CONCLUSIONES}

La susceptibilidad de un individuo a la periodontitis es el resultado de la interacción de múltiples influencias genéticas y medioambientales dentro de su carácter de enfermedad multifactorial.

La información genética nos puede ayudar a conocer la futura evolución de la enfermedad ante nuestro tratamiento y establecer protocolos individualizados. El conocimiento de los genotipos con más significación en la aparición y desarrollo de la periodontitis y su relación con determinados factores ambientales como el tabaco debe aumentar la habilidad del clínico en asignar un pronóstico y predecir la supervivencia del 
diente. Este conocimiento debe tenerse en cuenta para desarrollar un plan de tratamiento y establecer un protocolo de mantenimiento adecuado. El desarrollo de los test genéticos pueden ser útiles para identificar pacientes que tienen más riesgo de desarrollar la enfermedad, sufrir una recaída o perder dientes como consecuencia de la enfermedad. Como ya hemos visto, la utilidad de estos test tiene que ser validada para cada población o raza.

Estudios prospectivos que determinen los polimorfismos genéticos más relevantes y la aparición de tests más asequibles o accesibles para el clínico y paciente facilitaran la aplicación clínica de este emergente campo de investigación dentro de la periodoncia.

\section{SUMMMARY}

Periodontitis is a multifactorial disease in which the interaction of periodontal pathogens with the host immune defense machanism characterizes an inflamatory reaction that affects the attachment apparatus of the tooth. Multifactorial diseases normally involve complex genetic-enviromental interactions. The type of genetic variations described in these diseases are defined as polymorphisms. Different polymorphysm have been observed and normally the phenotypic consequences are limited.

In the first part of this review the evidence of association between Periodontitis and Genetics is analyzed. In the second part a special focus will be dedicated to research on polymorphysm and the clinical projection related to Periodontitis.

\section{KEY WORDS}

Periodontitis, susceptibility, genetic, polymorphism.

\section{BIBLIOGRAFÍA}

1. Hart TC, Kornman KS. Genetic factors in the pathogenesis of periodontitis. Periodontol 2000. 1997 Jun; 14:202-15. Review. No abstract available. PMID: 9567972 [PubMed - indexed for MEDLINE.

2. H ennig BJ, Parkhill JM, Chapple IL, Heasman PA, Taylor JJ. Association of a vitamin D receptor gene polymorphism with localized early-onset periodontal diseases. J Periodontol. 1999 Sep;70(9):1032-8.
3. Jeffcoat MK, Chesnut CH 3rd. Systemic osteoporosis and oral bone loss: evidence shows increased risk factors. J Am Dent Assoc. 1993 Nov; 124(11):49-56. Review.

4. Wactawski-Wende J, Grossi SG, Trevisan M, Genco RJ, Tezal M, Dunford RG, Ho AW, Hausmann E, Hreshchyshyn MM. The role of osteopenia in oral bone loss and periodontal disease. J Periodontol. 1996 Oct;67(10 Suppl): 1076-84. Review..

5. Ronderos M, Jacobs DR, Himes JH, Pihlstrom BL. Associations of periodontal disease with femoral bone mineral density and estrogen replacement therapy: cross-sectional evaluation of US adults from NHANES III. J Clin Periodontol. 2000 Oct;27(10):778-86.

6. Morrison NA, Qi JC, Tokita A, Kelly PJ, Crofts L, Nguyen TV, Sambrook PN, Eisman JA. Prediction of bone density from vitamin D receptor alleles. Nature. 1994 Jan 20;367(6460):284-7. Erratum in: Nature 1997 May $1 ; 387(6628): 106$.

7. Yamamoto K, Kobayashi T, Grossi S, Ho AW, Genco RJ, Yoshie H, De Nardin E. Association of Fcgamma receptor IIa genotype with chronic periodontitis in Caucasians. J Periodontol. 2004 Apr;75(4):517-22.

8. Deas DE, Mackey SA, McDonnell HT. Systemic disease and periodontitis: manifestations of neutrophil dysfunction. Periodontol 2000. 2003;32:82-104. Review.

9. Fujita T, Kantarci A, Warbington ML, Zawawi KH, Hasturk H, Kurihara H, Van Dyke TE CD38 expression in neutrophils from patients with localized aggressive periodontitis. J Periodontol. 2005 Nov;76(1 1):1960-5.

10. Lawton AR. IgG subclass deficiency and the day-care generation. Pediatr Infect Dis J. 1999 May;18(5):462-6. Review.

11. Albandar JM, DeNardin AM, Adesanya MR, Diehl SR, Winn DM Associations between serum antibody levels to periodontal pathogens and early-onset periodontitis. J Periodontol. 2001 Nov;72(1 1):1463-9.

12. Tangada SD, Califano JV, Nakashima K, Quinn SM, Zhang JB, Gunsolley JC, Schenkein HA, Tew JG. The effect of smoking on serum IgG2 reactive with Actinobacillus actinomycetemcomitans in early-onset periodontitis patients. J Periodontol. 1997 Sep;68(9):842-50.

13. Choi JI, Ha MH, Kim JH, Kim SJ. Immunoglobulin allotypes and immunoglobulin G subclass responses to Actino- 
bacillus actinomycetemcomitans and Porphyromonas gingivalis in early-onset periodontitis. Infect Immun. 1996 Oct;64(10):4226-30.

14. Deplazes P, Smith NC, Arnold P, Lutz H, Eckert J. Specific IgGl and IgG2 antibody responses of dogs to Leishmania infantum and other parasites.Parasite Immunol. 1995 Sep; 17(9):451-8.

15. Albandar JM, Muranga MB, Rams TE. Prevalence of aggressive periodontitis in school attendees in Uganda. J Clin Periodontol. 2002 Sep;29(9):823-31.

16. Houston LS, Lukehart SA, Persson GR, Page RC. Function of anti-Porphyromonas gingivalis immunoglobulin classes in immunized Macaca fascicularis. Oral Microbiol Immunol. 1999 Apr; 14(2):86-91.

17. Wilson ME, Bronson PM. Opsonization of Actinobacillus actinomycetemcomitans by immunoglobulin $\mathbf{G}$ antibodies to the $\mathrm{O}$ polysaccharide of lipopolysaccharide. Infect Immun. 1997 Nov;65(11):4690-5.

18. Durinovic-Bello I, Schlosser M, Riedl M, Maisel N, Rosinger S, Kalbacher H, Deeg M, Ziegler M, Elliott J, Roep BO, Karges W, Boehm BO. Pro- and anti-inflammatory cytokine production by autoimmune $\mathrm{T}$ cells against preproinsulin in HLA-DRB $1 * 04$, DQ8 Type 1 diabetes. Diabetologia. 2004 Mar;47(3):439-50.

19. Celenligil H, Kansu E, Ruacan S, Eratalay K, Caglayan G. Immunohistological analysis of gingival lymphocytes in adult periodontitis.J Clin Periodontol. 1990 Sep;17(8):542-8.

20. Amer A, Singh G, Darke C, Dolby AE. Association between HLA antigens and periodontal disease.Tissue Antigens. 1988 Feb;31(2):53-8.

21. Reinholdt J, Bay I, Svejgaard A Association between HLAantigens and periodontal disease. J Dent Res. 1977 Oct;56(10):1261-3.

22. Stein J, Reichert S, Gautsch A, Machulla HK. Are there HLA combinations typical supporting for or making resistant against aggressive and/or chronic periodontitis? J Periodontal Res. 2003 Oct;38(5):508-17.

23. Bonfil JJ, Dillier FL, Mercier P, Reviron D, Foti B, Sambuc R, Brodeur JM, Sedarat C. A "case control" study on the role of HLA DR4 in severe periodontitis and rapidly progressive periodontitis. Identification of types and subtypes using molecular biology (PCR.SSO). J Clin Periodontol. 1999 Feb;26(2):77-84.
24. Katz J, Goultschin J, Benoliel R, Brautbar C. Human leukocyte antigen (HLA) DR4. Positive association with rapidly progressing periodontitis. J Periodontol. 1987 Sep;58(9):607-10.

25. Cullinan MP, Sachs J, Wolf E, Seymour GJ. The distribution of HLA-A and -B antigens in patients and their families with periodontosis.J Periodontal Res. 1980 Mar;15(2): 177-84.

26. Saxen L, Koskimies S. Juvenile periodontitis-no linkage with HLA-antigens. J Periodontal Res. 1984 Sep;19 (5): 441-4.

27. Cascorbi I, Drakoulis N, Brockmoller J, Maurer A, Sperling K, Roots I. Arylamine N-acetyltransferase (NAT2) mutations and their allelic linkage in unrelated Caucasian individuals: correlation with phenotypic activity.Am J Hum Genet. 1995 Sep;57(3):581-92.

28. Meisel P, Timm R, Sawaf H, Fanghanel J, Siegmund W, Kocher T. Polymorphism of the N-acetyltransferase (NAT2), smoking and the potential risk of periodontal disease. Arch Toxicol. 2000 Aug;74(6):343-8.

29. Salvi GE, Brown CE, Fujihashi K, Kiyono H, Smith FW, Beck JD, Offenbacher S. Inflammatory mediators of the terminal dentition in adult and early onset periodontitis. J Periodontal Res. 1998 May;33(4):212-25.

30. Choi EM, Hwang JK Effects of Morus alba leaf extract on the production of nitric oxide, prostaglandin E2 and cytokines in RAW264.7 macrophages. Fitoterapia. 2005 Dec;76(7-8):608-13. Epub 2005 Oct 17.

31. di Giovine FS, Takhsh E, Blakemore AI, Duff GW. Single base polymorphism at -511 in the human interleukin-1 beta gene (ILl beta). Hum Mol Genet. 1992 Sep;1(6): 450 .

32. Kornman KS, di Giovine FS. Genetic variations in cytokine expression: a risk factor for severity of adult periodontitis.Ann Periodontol. 1998 Jul;3(1):327-38.

33. Kornman KS, Crane A, Wang HY, di Giovine FS, Newman MG, Pirk FW, Wilson TG Jr, Higginbottom FL, Duff GW. The interleukin-l genotype as a severity factor in adult periodontal disease.J Clin Periodontol. 1997 Jan;24 (1):72-7

34. Nieri M, Muzzi L, Cattabriga M, Rotundo R, Cairo F, Pini Prato GP. The prognostic value of several periodontal factors measured as radiographic bone level variation: 
a 10-year retrospective multilevel analysis of treated and maintained periodontal patients. J Periodontol. 2002 Dec;73(12):1485-93

35. Gore EA, Sanders JJ, Pandey JP, Palesch Y, Galbraith GM. Interleukin-1 beta +3953 allele 2: association with disease status in adult periodontitis. J Clin Periodontol. 1998 Oct;25(10):781-5.

36. McDevitt MJ, Wang HY, Knobelman C, Newman MG, di Giovine FS, Timms J, Duff GW, Kornman KS. Interleukin1 genetic association with periodontitis in clinical practice. J Periodontol. 2000 Feb;71(2):156-63.

37. Meisel P, Siegemund A, Dombrowa S, Sawaf H, Fanghaenel J, Kocher T. Smoking and polymorphisms of the interleukin-l gene cluster (IL-lalpha, IL-1beta, and IL-IRN) in patients with periodontal disease. J Periodontol. 2002 Jan;73(1):27-32.

38. Meisel P, Schwahn C, Gesch D, Bernhardt O, John U, Kocher T. Dose-effect relation of smoking and the interleukin-l gene polymorphism in periodontal disease. J Periodontol. 2004

39. McGuire MK, Nunn ME. Prognosis versus actual outcome. IV.The effectiveness of clinical parameters and IL-l genotype in accurately predicting prognoses and tooth survival. J Periodontol. 1999 Jan;70(1):49-56.

40. De Sanctis M, Zucchelli G Interleukin-l gene polymorphisms and long-term stability following guided tissue regeneration

41. Lopez NJ, Jara L, Valenzuela CY. Association of interleukin-l polymorphisms with periodontal disease.J Periodontol. 2005 Feb;76(2):234-43.

42. Cullinan MP, Westerman B, Hamlet SM, Palmer JE, Faddy MJ, Lang NP, Seymour GJ. A longitudinal study of interleukin-l gene polymorphisms and periodontal disease in a general adult population. J Clin Periodontol. 2001 Dec;28(12):1137-44.

43. Ehmke B, Kress W, Karch H, Grimm T, Klaiber B, Flemmig TF. Interleukin-1 haplotype and periodontal disease progression following therapy. J Clin Periodontol. 1999 Dec;26(12):810-3.

44. Anusaksathien O, Sukboon A, Sitthiphong P, Teanpaisan R. Distribution of interleukin-1beta(+3954) and ILlalpha(-889) genetic variations in a Thai population group. J Periodontol. 2003 Dec;74(12):1796-802.
45. Armitage GC, Wu Y, Wang HY, Sorrell J, di Giovine FS, Duff GW.Low prevalence of a periodontitis-associated interleukin-1 composite genotype in individuals of Chinese heritage. J Periodontol. 2000 Feb;71(2):164-71.

46. Lauener RP, Goyert SM, Geha RS, Vercelli D. Interleukin 4 down-regulates the expression of CDI4 in normal human monocytes. Eur J Immunol. 1990.

47. Jenkins $\mathrm{K}$, Javadi $\mathrm{M}$, Borghaei RC. Interleukin-4 suppresses IL-1-induced expression of matrix metalloproteinase-3 in human gingival fibroblasts. J Periodontol. 2004 Feb;75(2):283-91.

48. Michel J, Gonzales JR, Wunderlich D, Diete A, Herrmann JM, Meyle J. Interleukin-4 polymorphisms in early onset periodontitis. J Clin Periodontol. 2001 May;28(5):483-8.

49. Gonzales JR, Kobayashi T, Michel J, Mann M, Yoshie H, Meyle J. Interleukin-4 gene polymorphisms in Japanese and Caucasian patients with aggressive periodontitis.

50 . Al-Rasheed A, Scheerens H, Rennick DM, Fletcher HM, Tatakis DN. Accelerated alveolar bone loss in mice lacking interleukin-10.J Dent Res. 2003 Aug;82(8): 632-5.

51. Turner DM, Williams DM, Sankaran D, Lazarus M, Sinnott PJ, Hutchinson IV An investigation of polymorphism in the interleukin-10 gene promoter. Eur J Immunogenet. $1997 \mathrm{Feb} ; 24(1): 1-8$

52. Scarel-Caminaga RM, Trevilatto PC, Souza AP, Brito RB, Camargo LE, Line SR. Interleukin 10 gene promoter polymorphisms are associated with chronic periodontitis. J Clin Periodontol. 2004 Jun;31(6):443-8

53. Berglundh T, Donati M, Hahn-Zoric M, Hanson LA, Padyukov L. Association of the -1087 IL 10 gene polymorphism with severe chronic periodontitis in Swedish Caucasians. J Clin Periodontol. 2003 Mar;30 (3):249-54.

54. Holla LI, Fassmann A, Vasku A, Znojil V, Vanek J, Vacha J. Interactions of lymphotoxin alpha (TNF-beta), angiotensin-converting enzyme (ACE), and endothelin1 (ET-1) gene polymorphisms in adult periodontitis.J Periodontol. 2001 Jan;72(1):85-9.

55. Bascones A, Noronha S, Gomez M, Mota P, Gonzalez Moles MA, Dorrego MV. Tissue destruction in periodontitis: bacteria or cytokines fault?.Quintessence Int. 2005 Apr;36(4):299-306. Review. 


\section{AVANCES}

Volumen 20 - No 2 - Agosto 2008

56. Kurtis B, Tuter G, Serdar M, Akdemir P, Uygur C, Firatli E, Bal B. Gingival Crevicular Fluid Levels of Monocyte Chemoattractant Protein-1 and Tumor Necrosis Factor-Alpha in Patients With Chronic and Aggressive Periodontitis. J Periodontol. 2005 Nov; 76(11): 184955.

57. Ikezawa I, Tai H, Shimada Y, KomatsuY, Galicia JC,Yoshie $\mathrm{H}$. Imbalance between soluble tumour necrosis factor receptors type 1 and 2 in chronic periodontitis.J Clin Periodontol. 2005 Oct;32(10):1047-54.

58. Lin L, Pan YP, Yin LY. Study on the correlation of cytokine gene polymorphism with chronic periodontitis. Shanghai Kou Qiang Yi Xue. 2003 Dec; 12(6):456-9.

59. Donati M, Berglundh T, Hytonen AM, Hahn-Zoric M, Hanson LA, Padyukov L. Association of the -159 CD14 gene polymorphism and Q551R IL-4RA polymorphisms with severe chronic periodontitis in Swedish Caucasians. J Clin Periodontol. 2005 May;32(5):474-9.

60. Folwaczny M, Glas J, Torok HP, Mende M, Folwaczny C. Lack of association between the TNF alpha G -308 A promoter polymorphism and periodontal disease.J Clin Periodontol. 2004 Jun;31(6):449-53.

61. Vassalli P. The pathophysiology of tumor necrosis factors. Annu Rev Immunol. 1992; 10:41 1-52. Review.

62. Andres HH, Vogel RS, Tarr GE, Johnson L, Weber WW. Purification, physicochemical, and kinetic properties of liver acetyl-CoA:arylamine Nacetyltransferase from rapid acetylator rabbits. Mol Pharmacol. 1987 Apr;31(4):446-56.

62. Cattabriga M, Rotundo R, Muzzi L, Nieri M, Verrocchi G, Cairo F, Pini Prato G. Retrospective evaluation of the infuence of the interleukin-l genotype on radiographic bone levels in treated periodontal patients over 10 years. J Periodontol. 2001 Jun;72(6):767-73.

\section{CORRESPONDENCIA}

\section{Rodrigo-Gómez D}

Alvarfañez de Minaya, 2.

19001. Guadalajara

dperio@hotmail.com 